\title{
Vehicle interconnection metric and clustering protocol for improved connectivity in vehicular ad hoc networks
}

\author{
Samo Vodopivec ${ }^{*}$, Melita Hajdinjak, Janez Bešter and Andrej Kos
}

\begin{abstract}
Communication is the main driving force behind the emerging intelligent transportation systems, which are expected to make traveling safer, more ecological, and faster. The most challenging among all the different communication technologies that will be used are the direct vehicle-to-vehicle communications, because vehicles move with high speeds and in different directions. Apart from that, it is expected that vehicles should be able to organize themselves in an ad hoc network without any assistance from outside entities, such as road side units. To make the ad hoc network less dynamic and communications more reliable, vehicles with similar movement patterns can be grouped together in clusters. Clustering is a well-known method for organizing ad hoc networks and is used in mobile and wireless sensor networks, but with different constraints and goals, so new clustering solutions for vehicular ad hoc networks (VANET) have to be developed. In recent years, this has been a hot topic among researchers and many different clustering algorithms for VANET have been proposed. In this paper, we propose a new clustering metric for VANET, named vehicle interconnection metric, which is based on sending periodic beacons among vehicles and reflects the communication abilities between them. We also propose a new clustering algorithm whose primary goal is increased connectivity and lower number of disconnects. The working principle of this algorithm is also inverted compared to others and uses unneeded cluster head elimination instead of cluster head election. Mathematical analysis of memory usage and communication overhead are provided, predicting low-resource usage. Simulation results, obtained with the ns-3 network simulator and the SUMO vehicle movement simulator, have confirmed the analysis and expected performance in terms of cluster head duration, number of connectivity losses and role switches.
\end{abstract}

Keywords: Clustering; Vehicular ad hoc network; VANET; Intelligent transportation systems; Vehicle interconnection; Connectivity

\section{Introduction}

Intelligent transportation systems (ITS) are one of the most promising emerging technologies. They are regarded as the key to safer, faster, and more ecological transportation of the near future. The main motivation comes from the safety field where numerous lives are expected to be saved each year due to the ability of vehicles to prevent accidents from happening or at least lowering their toll. This, as a consequence, also prevents congestions which cause traffic accidents, allowing the traffic to flow more smoothly, shortening the travel time,

\footnotetext{
* Correspondence: Samo.Vodopivec@fe.uni-lj.si

Faculty of Electrical Engineering, University of Ljubljana, Tržaška cesta 25, Ljubljana SI-1000, Slovenia
}

and lowering the fuel usage. Even more services that will make traveling more user-friendly are planned, for example, real-time route optimization according to traffic situation, cooperative adaptive cruise control, free parking space search, and infotainment.

One of the most prominent driving forces for ITS is the fact that communications are using many different technologies such as 3G/4G, WiFi, dedicated short-range communications (DSRC) [1] and others. The ubiquitous communications [2] are important because they will allow fusing of different data sources to provide new services with the desired usability, reliability, and availability.

Vehicle-to-vehicle (V2V) communications are one of the most challenging. They are the only type of communication

\section{穴}


that will be available all the time, making it suitable for the most important services such as collision prevention and other safety features. On the other hand, data dissemination between vehicles can be a difficult task due to their high mobility and relatively low communication range on the 5.9-GHz band where DSRC is defined [1]. To lessen the effect of high mobility, vehicles with a similar movement pattern can be grouped together in clusters.

Clustering is a process of grouping elements with similar properties in clusters and can be used in many different fields. One of the uses is also in the wireless ad hoc communications where clustering is used to make the network appear more stable on the logical level, providing solid foundation for upper layer protocols. Wireless ad hoc networks are further divided into different categories, such as mobile, wireless sensor, and vehicular, each category with its own specifics. These specifics are the reason that algorithms designed for one category do not scale very well when used in another. For vehicular ad hoc networks (VANET), the specifics are the high speed of nodes that are obliged to move according to traffic regulations and the relatively unconstrained energy and computing resources.

Designing a good clustering algorithm for VANET is a challenging task due to the variety of situations where vehicles are present. Highways and city centers, congestions, and empty roads, etc. all influence the communication parameters, but clustering should provide decent performance for all of them. However, because clustering is one of the fundamental parts of $\mathrm{V} 2 \mathrm{~V}$ communications, dependence on other services such as positioning should be avoided to prevent compromising the communications in case of unavailability of the other services.

Many clustering algorithms for VANET have been proposed in the recent years [3], of which the majority are Global Positioning System (GPS) based, with the main goal of stabilizing the clusters and minimizing the number of cluster heads. We took a different approach and set the goal of increasing connectivity and lowering the number of disconnects, thus providing better usability of the V2V communications. We designed a new clustering metric based on beacon frames sent between vehicles that exposes the vehicles' similarity in movement from the communication point of view. This metric is used in the proposed clustering algorithm, which requires each node to be connected with two cluster heads to improve the connectivity. The working principle of this algorithm is also inverted, so unneeded cluster head elimination is used instead of cluster head election.

This paper offers the following original contributions. Firstly, we propose an improved vehicle interconnection metric that exposes the similarity of vehicles' movement pattern in time without using positioning services. Secondly, we propose a tightly coupled variant of the clustering protocol based on the interconnection metric. Thirdly, we provide a mathematical analysis of the clustering protocol's overhead. Fourthly, we prove the overhead analysis with simulation results. Last but not the least, we compare the performance of our clustering solution to the MOBIC [4] clustering protocol.

\section{Related work}

A general overview of VANET is presented in [5]. The paper presents the aspects related to this field in order to help researchers and developers understand and distinguish the main features surrounding VANET in one solid document without the need to do in-depth research by themselves.

The VANET clustering is a hot topic among researchers, and in recent years more than 20 scientific papers have been published [3]. This trend is not showing any slowdowns due to the expected growth of the ITS deployments in the next decade.

The majority of the proposed clustering algorithms for VANET depend on some sort of positioning service, usually on a global navigation satellite system (GNSS) such as GPS or its alternatives. But it is a known fact that positioning services are not available everywhere, and even if they are, their accuracy varies significantly $[6,7]$. The problematic areas are especially parking structures and tunnels, bridges, city centers with narrow streets and high buildings, narrow valleys, etc. GNSS is also vulnerable to spoofing and jamming attacks $[8,9]$ that can prevent its correct operation. To avoid the loss of communications in case of unavailable or unreliable positioning services, clustering and other low-level communications should function independently.

The communications in VANET take place on the 5.9$\mathrm{GHz}$ wireless band where the DSRC [1] and the newer IEEE 1609 [10] family of standards, named wireless access in vehicular environments (WAVE), are defined. Due to limited bandwidth, which tops at a maximum of $27 \mathrm{Mbps}$, and other quality of service (QoS) constraints, special care has to be taken to prevent channel congestion as important services, such as collision avoidance, could be compromised. Clustering is one of the possible methods for lowering the overhead as it organizes the network and lowers the amount of broadcasts and retransmits that can otherwise clog up the network.

The paper [11] proposes a robust criticality-based clustering algorithm (CCA) based on the concept of the network criticality. Network criticality is a global metric on an undirected graph, which quantifies the robustness of the graph against environmental changes such as topology. Authors localize the notion of network criticality and merge it with a universal link measure, link expiration time (LET), to derive a distributed multi-hop clustering algorithm. 
A novel, mobility-based clustering scheme, which forms clusters using the Affinity Propagation algorithm in a distributed manner, is proposed in [12]. The algorithm considers node mobility during cluster formation, produces clusters with high stability and is also robust enough to channel error and exhibits reasonable overhead.

In [13] the Vehicular Multi-hop algorithm for Stable Clustering (VMaSC) is introduced. It is a novel clustering technique based on choosing the node with the least mobility calculated as a function of the speed difference between neighboring nodes.

Virtual Forces Vehicular Clustering (VFVC) [14] is a new clustering algorithm that creates stable clusters in urban environments where the mobility pattern of vehicles is more spatial. The algorithm uses combined metrics produced by a vehicle's position, geometry, relative velocity, and vehicle's lane in order to assign virtual forces among them and create clusters.

A research of the novel Multi-resolution Relative Speed Detection (MRSD) model to improve the clustering algorithm in VANET without using GPS is presented in [15]. It uses the moving average convergence divergence, the momentum of the received signal strength, and artificial neural networks to estimate the motion state and the relative speed of a vehicle, based purely on received signal strength.

Recently, two fuzzy logic-based clustering algorithms have been presented. The first [16] proposes a novel cluster head selection criteria where cluster heads are selected based on their relative speed and distance from vehicles, within their neighborhood. The maintenance phase is adaptable to drivers' behavior on the road and has a learning mechanism for predicting the future speed and position of all cluster members using fuzzy logic inference system. The second algorithm [17] proposes a novel user-oriented fuzzy logic-based $k$-hop distributed clustering scheme for VANETs that takes into consideration the vehicle passenger preferences.

In [18], authors study the QoS performance of different routing schemes for vehicular networks. They propose a double cluster head routing solution as a compromise between end to end delay, packet loss, and energy consumption.

A virtual mobile clustering-based solution that improves connectivity on a highway is presented in [19]. The authors introduce the average speed election factor and vector velocity variable to differ between vehicles driving in opposite directions in order to reduce the fast connection and disconnection events that usually overload the network and reduce the clustering performance.

The node degree, the available resource of candidate cluster heads, and the velocity difference between them is jointly considered when selecting a cluster head in the algorithm presented in [20]. The proposed cluster switching scheme stresses the QoS requirements of both delaysensitive services as well as throughput-sensitive services; by defining utility functions for accessing various clusters, the optimal destination cluster can be obtained.

The paper [21] proposes a cluster-based algorithm which uses Prim's algorithm to implement a minimum spanning tree when forming clusters. This algorithm takes into consideration the QoS of intra-cluster communication by calculating a maximum cluster size for every cluster beyond which the quality of communication within the cluster is not acceptable. The clusters are also formed only for the purpose of information dissemination and are dissolved immediately after the information is relayed, so no resources are lost for cluster management during the inactive phase.

Splitting the network into 1-hop clusters of different sizes is the goal of the algorithm presented in [22]. Instead of considering the node degree, the proposed protocol relies on the mobility characteristics and correlation properties among the nodes within the same radio coverage. By exploiting information provided by Cooperative Awareness Messages that are broadcast periodically by each vehicle, an estimated connectivity time is computed and used to select a proper cluster head to form stable and long-lived clusters.

\section{The vehicle interconnection metric}

The metric we propose highlights the similarity of vehicle movement in space and time and produces the foundation for stable clustering. It works in a distributed fashion and is based on periodic beacon messages between vehicles. For simplicity reasons, we assume that the beacon period is constant and equal for all the vehicles.

Each vehicle $i$ maintains two list entries for every neighboring vehicle $j$. List entries $m_{i}(j)$ and $b_{i}(j)$ store the metric value for the vehicle $j$ and the number of consecutive beacon missed receptions from vehicle $j$, respectively, as perceived by the vehicle $i$. Every list entry is stored as an 8 bit unsigned integer with values that range from 0 to 255 . The values are updated every beacon period, depending on the reception or lack of beacon reception from the nearby vehicle.

At the beginning, all the values in both lists are set to zero. Upon successful reception of a beacon from the vehicle $j$, the metric value $m_{i}(j)$ is rewarded by increasing the value by $1(1)$. At the same time, the entry in the $b_{i}(j)$ is reset to zero (2), denoting no consecutive beacon misses.

$$
\begin{aligned}
& m_{i}(j)=\left\{\begin{array}{cc}
m_{i}(j)+1, & m_{i}(j)<255 \\
255, & \text { otherwise }
\end{array}\right. \\
& b_{i}(j)=0
\end{aligned}
$$

Before sending its own beacon, a vehicle enters the metric maintenance routine. Here, all the vehicles $j$ from the lists $m_{i}(j)$ are processed and if, according to $b_{i}(j)$, there have been beacon misses, the metric is penalized 
by exponential subtraction (3), which lowers the interconnection value for that vehicle. If the metric value reaches zero, then the vehicle is removed from both lists; otherwise, the value $b_{i}(j)$ is increased by $1(4)$. After this process is finished, the vehicle sends its beacon.

$$
\begin{aligned}
& m_{i}(j)=\left\{\begin{array}{cc}
m_{i}(j)-2^{b_{i}(j)-1}, & b_{i}(j)>0 \\
m_{i}(j), & \text { otherwise }
\end{array}\right. \\
& b_{i}(j)=b_{i}(j)+1
\end{aligned}
$$

In the first proposal of this metric, we investigated divide-by-two as the penalty function, but it turned out to be too aggressive. So the function was changed to exponential subtraction where the exponent is the number of consecutive beacon misses. In this case, the penalty is small in the first step but increases quickly, thus forgiving erroneous beacon misses due to collision or random channel noise.

As the metric is not symmetrical, the value $m_{i}(j)$ is not necessarily the same as $m_{j}(i)$. This comes from the design and does not present a limitation because the values are usually, in normal communication conditions, more or less the same.

The metric logically separates vehicles in the area into groups with a similar mobility pattern as shown in Figure 1. The higher the metric value, the longer the vehicles were able to communicate reliably. So they had to be in close vicinity for a longer period of time, regardless if they are slowly moving trucks or speeding sports cars. The number of different groups is not predetermined but depends on the movement pattern of the vehicles in the area. There might be a few groups on a highway in normal driving conditions but only one in the case of congestion.

The term 'group', which is being discussed here, can only be used by an outside identity that would have access to the metric tables from all the vehicles in the area. But due to the decentralized concept of the metric, the vehicles themselves know very little about the other groups in their neighborhood. Every vehicle can only distinguish between vehicles which are more likely to be in the same group and the vehicles that are less likely to be in it. But for the proposed clustering algorithm, this information is sufficient.

\section{Clustering}

The main goal of our clustering solution is the superior connectivity that minimizes the network interrupts caused by unpredictable vehicle movement. To achieve this goal, a few rarely used concepts have been investigated, some of them, according to the best of our knowledge, for the first time in VANET clustering.

Multi-homing is a feature known from the SCTP protocol [23] that allows a host to be accessible via multiple different routes. This provides redundant connections and thus increases the communication reliability. A similar concept is used in our clustering algorithm where each vehicle, regardless of its type, strives to be connected with two cluster heads at any given time. This lowers the probability of a vehicle being disconnected from both cluster heads at the same time, thus improving the connectivity. A similar concept has already been investigated for VANET data routing in [18].

The other novelty we propose is the cluster building principle which is inverted compared to others. Instead of electing a cluster head among the nodes, each node is a cluster head by default and switches to cluster node state when it becomes unnecessary (and vice versa). Because the algorithm works in a distributed fashion and each node decides about the switch independently, the complex and slow cluster reorganization processes known from other algorithms are avoided.

The last rarity we investigate is the location service independence. The majority of the proposed clustering algorithms use some form of location services, usually GPS, to cluster vehicles according to their position and/ or movement. But these services are not available everywhere and their accuracy and reliability varies significantly [6,7]. They are also vulnerable to spoofing and jamming attacks [9]. It is highly undesirable that a service like this influences the performance of the communication network, so our proposed solution avoids using location services altogether.

In the next paragraphs, the following definitions are used to describe the algorithm:

- A suitable cluster head, from a node's point of view, is a cluster head with an interconnection metric value higher than 10 . In case of more such cluster heads, the ones with the higher metric value are

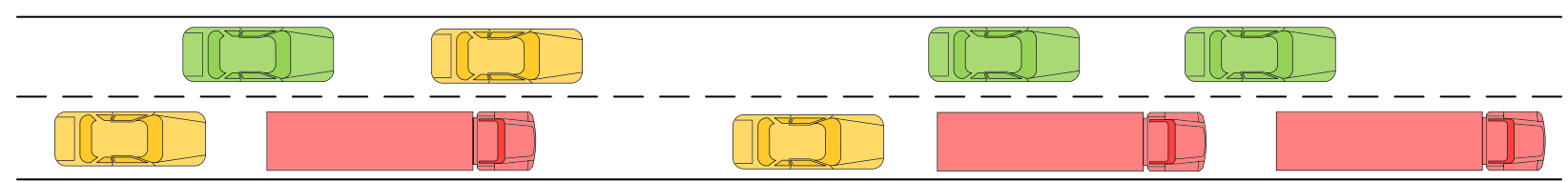

Figure 1 Logical separation of vehicles into groups as performed by the interconnection metric. Three different vehicle groups are shown, each in its own color. 
preferred. The threshold value 10 prevents the formation of unstable clusters but, on the other hand, allows the clustering process to start early - after a node is present for at least 10 beacon periods.

- A fully connected node is a node that is connected to two suitable cluster heads. If a node is disconnected or connected to only one suitable cluster head, then it is considered as not fully connected.

In addition to list entries $m_{i}(j)$ and $b_{i}(j)$ that are used for the metric, the list entry $c_{i}(j)$ keeps the cluster head state of the vehicle $j$ and is used by the clustering algorithm for keeping track of neighboring vehicles status.

\section{Cluster head elimination}

Due to the nature of our algorithm, where cluster head is the default node state, superfluous cluster heads need to give up their role and become cluster members. This process should disturb the network as little as possible to avoid unnecessary connectivity issues.

By definition, each node should be connected to two cluster heads, so the probability is quite high that a node will stay connected to at least one of them. A node is free to switch the role anytime, but the algorithm tries to minimize the probability of two nearby cluster heads switching their state at the same time.

The first constraint that has to be met before a node can switch the role is the availability of at least two suitable cluster heads in its 1-hop vicinity. This is the most important constraint because there might be other cluster heads in the vicinity that are unsuitable for clustering, for example when driving in the opposite direction.

The next constraint is the network split prevention. The switching node checks its cluster heads for connectivity between them. If their cluster heads are not connected one to another, then the network could split, disabling the connectivity between both parts. This is avoided by preventing the cluster head to give up its role.

The minimum degree of the cluster head is the last constraint that needs to be fulfilled before switching roles is allowed. To minimize the effect of switching on the network, the cluster head is allowed to switch its state only if it has the lowest degree in the neighborhood. If two cluster heads have the same degree, then the switching is postponed until the situation changes. This implies that there might be more cluster heads in the network than needed at the given moment, but on the other hand it also prevents two neighboring nodes from switching states simultaneously, which is in our opinion more important.

When the switch to cluster member occurs, the node informs its surrounding vehicles via the beacon message as there are no explicit notifications about the change. Upon detecting this change, surrounding nodes connect to other cluster heads or, in rare cases, one of them switches to cluster head state.

\section{Cluster head creation}

Although each vehicle begins its journey as a cluster head, it is expected that it will give up this role sooner or later and become a cluster member. But as a result of vehicle movement, topological changes in the network might require a cluster member to switch back to the cluster head state. The unavailability of the suitable cluster heads in its 1-hop vicinity is the reason for a node to switch to cluster head state.

When a node misses two consecutive beacons from one of its cluster heads, it presumes that it is disconnected and starts looking for a new suitable cluster head. If such a cluster head is found, then the node joins it and stays in cluster member state. But if a suitable cluster head is not found, then the node switches its state to cluster head. To avoid oscillations, this node is prevented to switch back to cluster member state for the next two beacon periods.

\section{Intercluster communication}

An essential part for multi-hop VANET communication is intercluster communication, and many clustering algorithms fail to address this challenge while striving to minimize the number of cluster heads and maximize their lifetime. The majority of the proposed algorithms create non-overlapping clusters and as a consequence, cluster heads are unable to communicate directly one to another. This limitation is then handed over to the routing protocol or solved by defining cluster gateways. These gateways are nodes in the communication range of two or more cluster heads, but their behavior is commonly quite undefined and unpredictable which affects the link stability. As shown in Figure 2, there might be more possible cluster gateways between two cluster heads, but usually there is no defined rule on what basis to choose the gateway in any specific moment or for a specific task. Our clustering algorithm avoids this weakness by defining the direct cluster head to cluster head connectivity as shown in Figure 3. Every node, including the cluster heads themselves, requires connectivity with two other cluster heads, so no explicit gateways are needed. This implies a larger number of cluster heads compared to other algorithms, but on the other hand it simplifies network management and provides better scalability.

\section{Resource usage and protocol overhead}

The proposed algorithm has a very modest resource usage. The data structures $m_{i}(j), b_{i}(j)$, and $c_{i}(j)$ use 1 byte each per vehicle. Additional 4 bytes per vehicle are used 


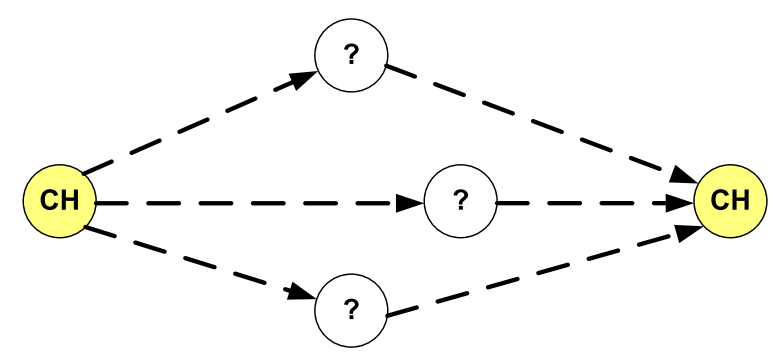

Figure 2 Data-forwarding dilemma in a usual clustering scenario without explicitly defined clustering gateways.

for storing the node id, making it 7 bytes all together. So even in the case of a huge amount of vehicles in a small area, for example, in a large multi-level parking building, the data structures would consume at most a few kilobytes of memory. However, in this case, the channel bandwidth and packet collisions would be a much bigger problem anyway. Also, all the used mathematic operations are integer based. This allows the protocol to be implemented on low-cost microcontrollers with limited resources, which is an advantage for early-stage ITS deployments and also a welcomed feature for the future.

Each beacon packet includes MAC, IP, and UDP headers with sizes of 58, 20, and 8 bytes, 86 bytes all together (5). The fixed part of the payload consists of 4 bytes MY_ID, 1 byte cluster head status CH_STAT, and two 4 bytes cluster heads ids, $\mathrm{CH} \mathrm{ID}_{1}$ and $\mathrm{CH} \mathrm{ID}_{2}$. Additionally, cluster heads also append the list of nodes they serve, so we need additional 4 bytes for each cluster member. The size of the beacon in bytes is defined in (6):

$$
\begin{aligned}
S_{\mathrm{HDR}}= & S_{\mathrm{MAC}_{\mathrm{H}} \mathrm{DR}}+S_{\mathrm{IP}_{\mathrm{H}} \mathrm{DR}}+S_{\mathrm{UDP}_{\mathrm{H}} \mathrm{DR}} \\
= & 58+20+86 \text { bytes } \\
S_{\mathrm{BEACON}}= & S_{\mathrm{HDR}}+S_{\mathrm{MY}_{\mathrm{ID}}}+S_{\mathrm{CH}_{\mathrm{STAT}}}+S_{\mathrm{CH}_{\mathrm{ID}} 1} \\
& +S_{\mathrm{CH}_{\mathrm{ID}} 2}+4 \times N_{\mathrm{MEMBERS}} \\
= & 86+4+1+4+4+4 \\
& \times N_{\mathrm{MEMBERS}} \\
= & 99+4 \times N_{\mathrm{MEMBERS}} \text { bytes }
\end{aligned}
$$

It is clearly visible from the Equation 6 that the minimum beacon size is 99 bytes when the variable part is

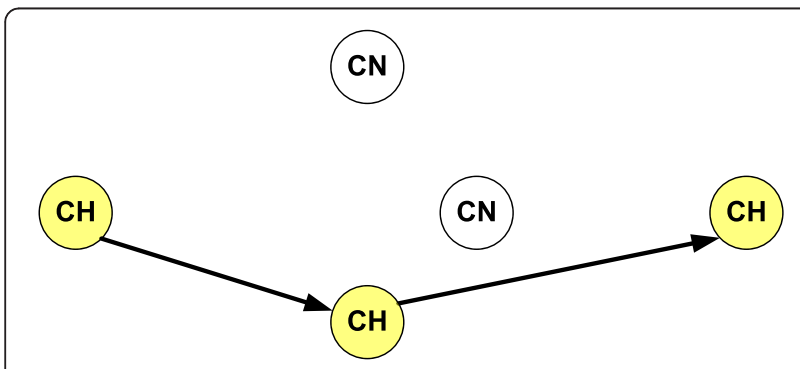

Figure 3 Data forwarding with direct cluster head to cluster head communication as proposed in the algorithm. not present (in case of cluster member). This is also the most frequently used size in a usual network as there are more cluster members than cluster heads.

Concerning the network as a whole, the lower bound of 99 bytes per beacon per node can only be achieved when all the nodes are unable to cluster with any other node. This can happen if the network is extremely sparse, for example, during the night. The upper bound for the average beacon size per node (7) is achieved, when all the vehicles in the network are fully connected, e.g., when each vehicle is connected to two cluster heads. This is more probable in dense networks where the distance between nodes is smaller. In this case, each node in the network is present on the service list of these two cluster heads, adding 4 bytes each, so 8 bytes of additional overhead per node.

$$
S_{\mathrm{BEACON}_{\mathrm{MAX}}}=99 \text { bytes }+2 \times 4 \text { bytes }=107 \text { bytes }
$$

Therefore, the average beacon size per node is somewhere between 99 and 107 bytes, depending on the network density and independent of the number of nodes. The predictable and upper bound-limited average beacon size is very important as it allows the network to scale better at higher loads. The beacon frame overhead can be further reduced by dropping IP and UDP protocols and using MAC frames only, but this would complicate the clustering algorithm as some features of the dropped protocols would have to be implemented separately.

Comparing the average beacon size with APROVE [12], our algorithm performs as well as APROVE in sparse, disconnected networks where both beacon sizes are the same. But in dense, connected networks, the difference can be up to and beyond $100 \%$ in favor of our algorithm. According to the protocol overhead analysis in [12], where APROVE is proven to be superior compared to MOBIC, consequently our algorithm also outperforms MOBIC in protocol overhead.

\section{Simulation}

Both clustering algorithms, the one proposed here and MOBIC, were implemented in the ns-3 network simulator [24], the successor of the very popular and renowned ns-2. The simulation was performed using 802.11p MAC on $5.9 \mathrm{GHz}$ with a constant transmission rate of $27 \mathrm{Mbps}$. The transmission range was set to $50,100,150,200,250$, and $300 \mathrm{~m}$ in different simulation runs. The beacon period for both algorithms was set to $1 \mathrm{~s}$.

The SUMO [25] simulator was used for vehicle movement data generation. It is a microtraffic simulator and provides information about the movement and position of every vehicle individually, which is a key factor for more 
realistic network simulation results. The integration between SUMO and ns-3 was done with the help of Ovnis [26].

To approximate a realistic vehicle movement, the traffic on a $5-\mathrm{km}$ road segment from Ljubljana to Medvode was simulated, as shown in the Figure 4. This segment includes roads with speed limits of 50,60 , and $90 \mathrm{~km} / \mathrm{h}$ and a traffic light at the end. Data from the loop detectors was used to approach closely to the authentic movement pattern of vehicles. Although this road is a two-lane, two-way road and the vehicles in each lane rarely change their order, the clustering process gets triggered because of changing vehicle density and speed due to congestions.
From our observations, this scenario provides a higher burden on the clustering algorithm than the usual highway scenarios.

Data from two different time slots in 1 day has been selected for the simulation. One of them represents the traffic flow under normal traffic conditions and the other under heavily increased traffic conditions.

Five vehicle categories have been defined with different acceleration and top speed parameters. The tendency of $13 \%$ of the vehicles is to drive $10 \%$ below the speed limit and $33 \%$ of the vehicles to drive $10 \%$ above the speed limit. The remaining $54 \%$ of the vehicles obey the speed

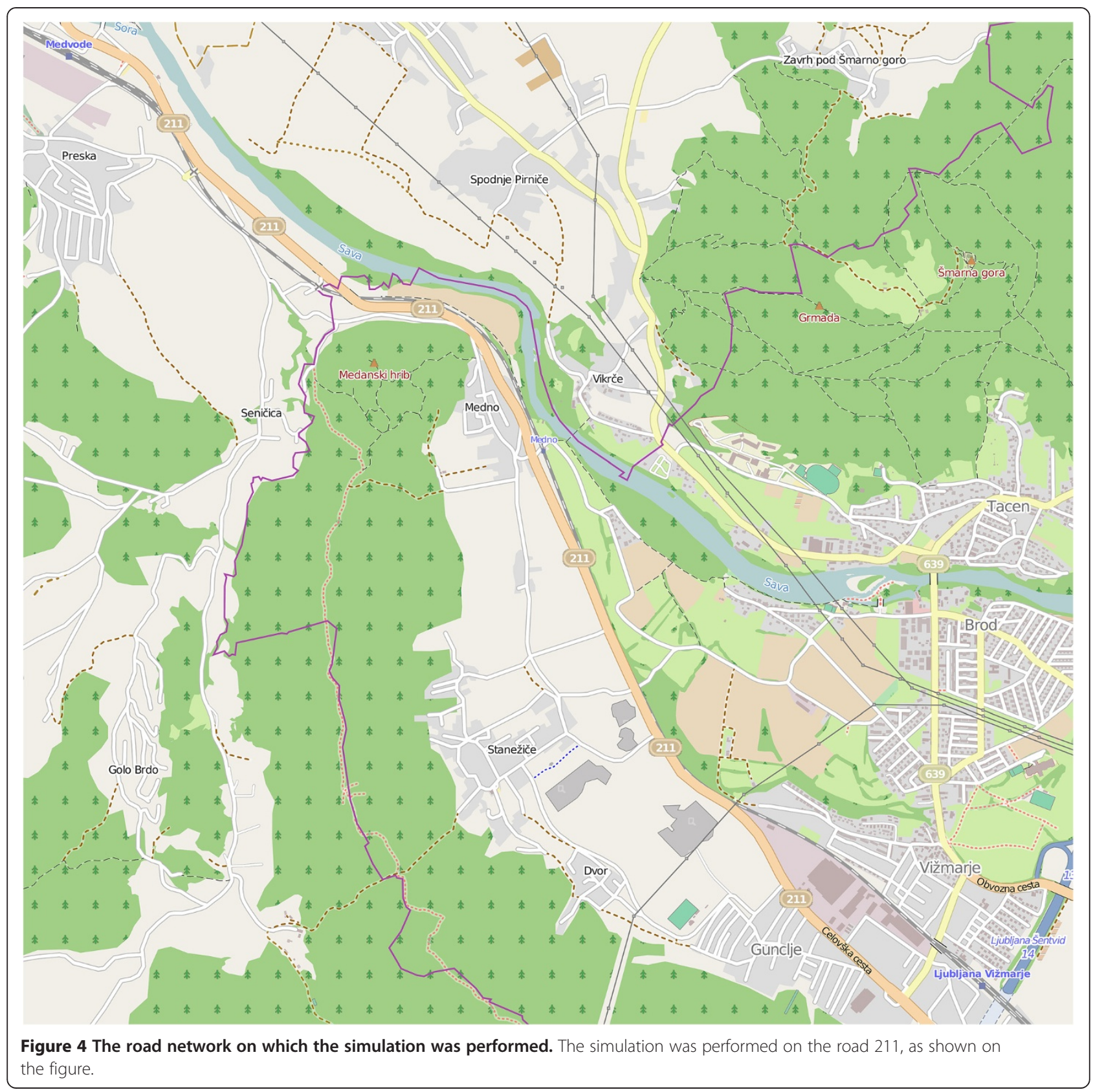


limits. These different types of vehicles create microgroups and enter additional dynamics into the scenarios when the groups split and merge.

The evaluation of both algorithms is done using the following metrics:

- The average cluster head duration shows how long a node stays in the cluster head state. The longer the time, the more stable the clusters. Because our algorithm strives to connect a node to two cluster heads instead of one, as in the case of MOBIC, the resulting values are divided by two to allow for a fair comparison.

- The average number of full connectivity loss per node shows how many times a node gets disconnected from the network. This happens when a node loses the connectivity to all of its cluster heads and needs to reconnect. A change of one cluster head in our algorithm is not interpreted as full connectivity loss, because the node stays connected to the other cluster head. Because the connectivity is one of the main goals of our algorithm, this metric plays an important role.

- The average number of role switches per node shows how many times a node switches its role from cluster head to cluster member and vice versa. This relates to cluster stability and the lower the number, the more stable the clusters. Because all the nodes in our algorithm begin as cluster heads, the numbers are not directly comparable to MOBIC, so the results are only compared between different runs of our algorithm.

- The protocol overhead was also measured for our algorithm to check the validity of the mathematical proof. This also exposes the influence of different vehicle densities on the protocol overhead.

Each simulation scenario was run for $40 \mathrm{~min}$, and the last $30 \mathrm{~min}$ were used for the performance measurements. This allowed the network to stabilize before taking the measurements, avoiding the oscillations that might have been present at the beginning. All the vehicles are removed from the simulation once they reach their final destination, and there are no loops that would allow a vehicle to stay in the simulation for a longer time.

\section{Results and discussion}

In all the simulation runs with the same traffic load, the same number of vehicles took part. In a normal load scenario, 557 vehicles were present on the roads and 939 vehicles in a heavily increased scenario.

During the analysis of the results, we observed that the results for $200 \mathrm{~m}$ and longer communication ranges are practically the same. This is the consequence of the signal propagation limitations, as the receiving signal was too weak to be received by more distant nodes. This observation was verified by the Kolmogorov-Smirnov test for assessing whether two samples were drawn from the same population [27]. We found that the data from our simulation scenarios, either of heavily increased or normal traffic conditions, follows the same probability distribution when the transmission range is set to $150 \mathrm{~m}$ or more. The Kolmogorov-Smirnov test gave $p$ values higher than 0.30 for all the observed properties/metrics. The distributions of the changes of only one cluster head in normal traffic conditions are shown in Figure 5. Due to this observation, the results for communication ranges above $200 \mathrm{~m}$ have sometimes been omitted from the presentation.

Except for the normal traffic density with the shortest communication range of $50 \mathrm{~m}$, our algorithm outperforms MOBIC in terms of the average cluster head duration. The improvement varies and reaches up to $20 \%$ as shown in Figure 6. The deviations at the shorter communication ranges are the consequence of different working principles. Because all the nodes in our algorithm start as cluster heads and the communication range is short, less of them switch to the cluster member state. But in high traffic load, the opposite can be observed - because traffic jams happen and the communication range is short, the nodes need to switch their states more often, lowering the cluster stability. Moreover, in contrast to the MOBIC algorithm, our algorithm gives equally distributed cluster head durations at the shortest communication range of $50 \mathrm{~m}$ in both normal and heavily increased traffic conditions. The Kolmogorov-Smirnov test gives a $p$ value of 0.18 . The probability distributions of cluster head duration in both traffic conditions are shown in Figure 7.

Equal distributions are also confirmed when changes of one cluster head in normal, and heavily increased traffic conditions at a transmission range of $50 \mathrm{~m}$ are compared. The Kolmogorov-Smirnov test gives a $p$ value of 0.24 . More importantly, for all transmission ranges and both traffic conditions, changes of one cluster head most likely follow the beta probability distribution with stretched support. This conclusion results from the $\chi^{2}$ goodness-of-fit test [22], in which we considered a wide range of hypothesized null distributions (i.e., Poisson, binomial, negative binomial, normal, gamma, lognormal, Weibull, beta; geometric, exponential, chi-squared, uniform). For instance, the number of changes of one cluster head in heavily increased traffic conditions and a transmission range of $100 \mathrm{~m}$ follows the beta distribution with shape parameters 0.91 and 1.94 at a significance level of 0.01 . The corresponding histogram with the beta distribution curve with stretched support is shown in Figure 8.

The connectivity improvement, to which our clustering algorithm is striving to, can be clearly observed in 


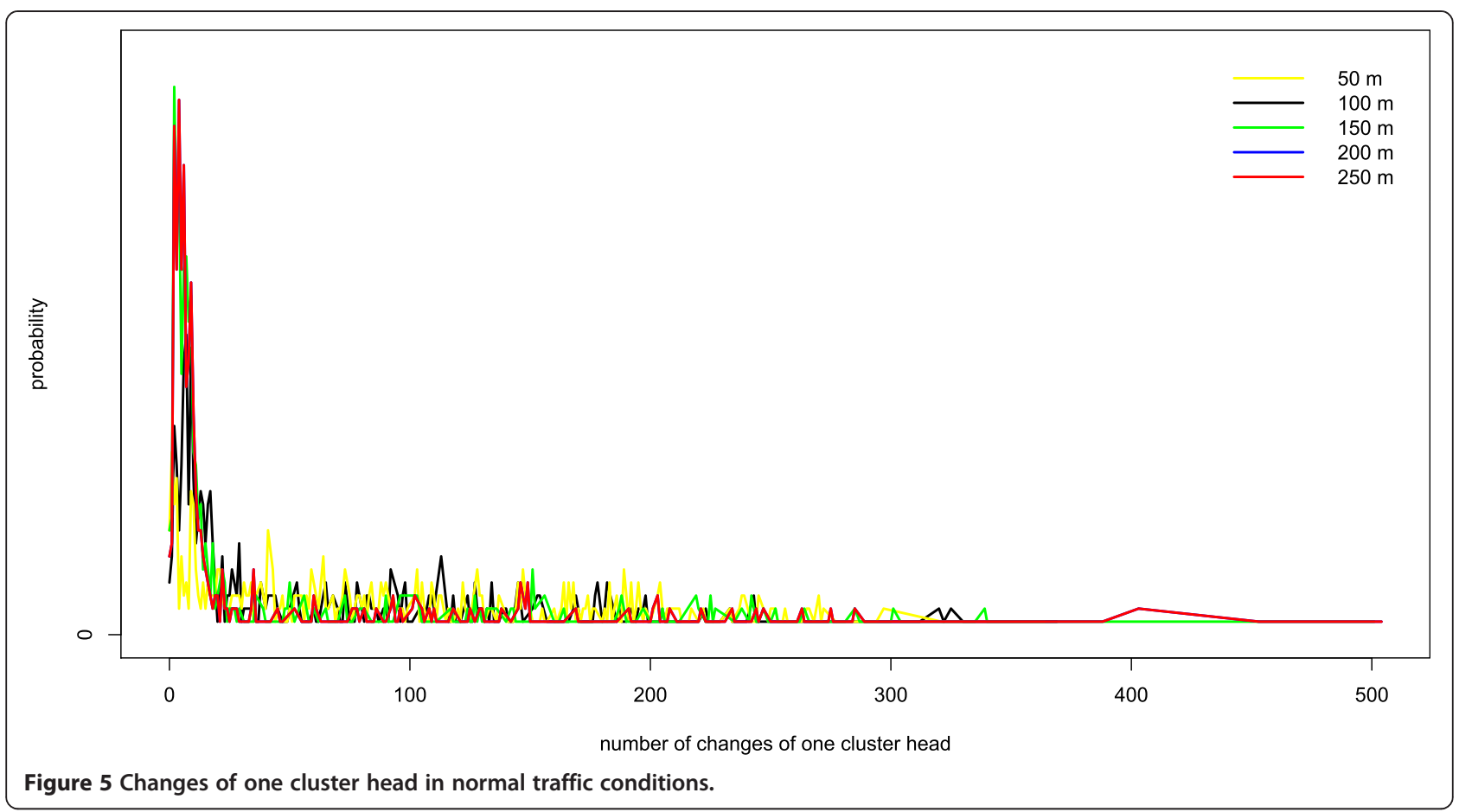

Figure 9. The number of cases in which a node is completely disconnected from the network is even up to three times lower when using our clustering algorithm instead of MOBIC. Both communication range and vehicle density affect the connectivity in the same way in both algorithms - increased communication range improves connectivity and increased vehicle density lowers it.
The average number of role switches per node is shown in Figure 10. It is clearly observable that less switching occurs when the communication range is longer, because the vehicles in the vicinity are perceived as more stable. More role switches in case of heavy traffic are also not surprising because more vehicles induce a higher dynamic. From the $x^{2}$ goodness-of-fit test, we

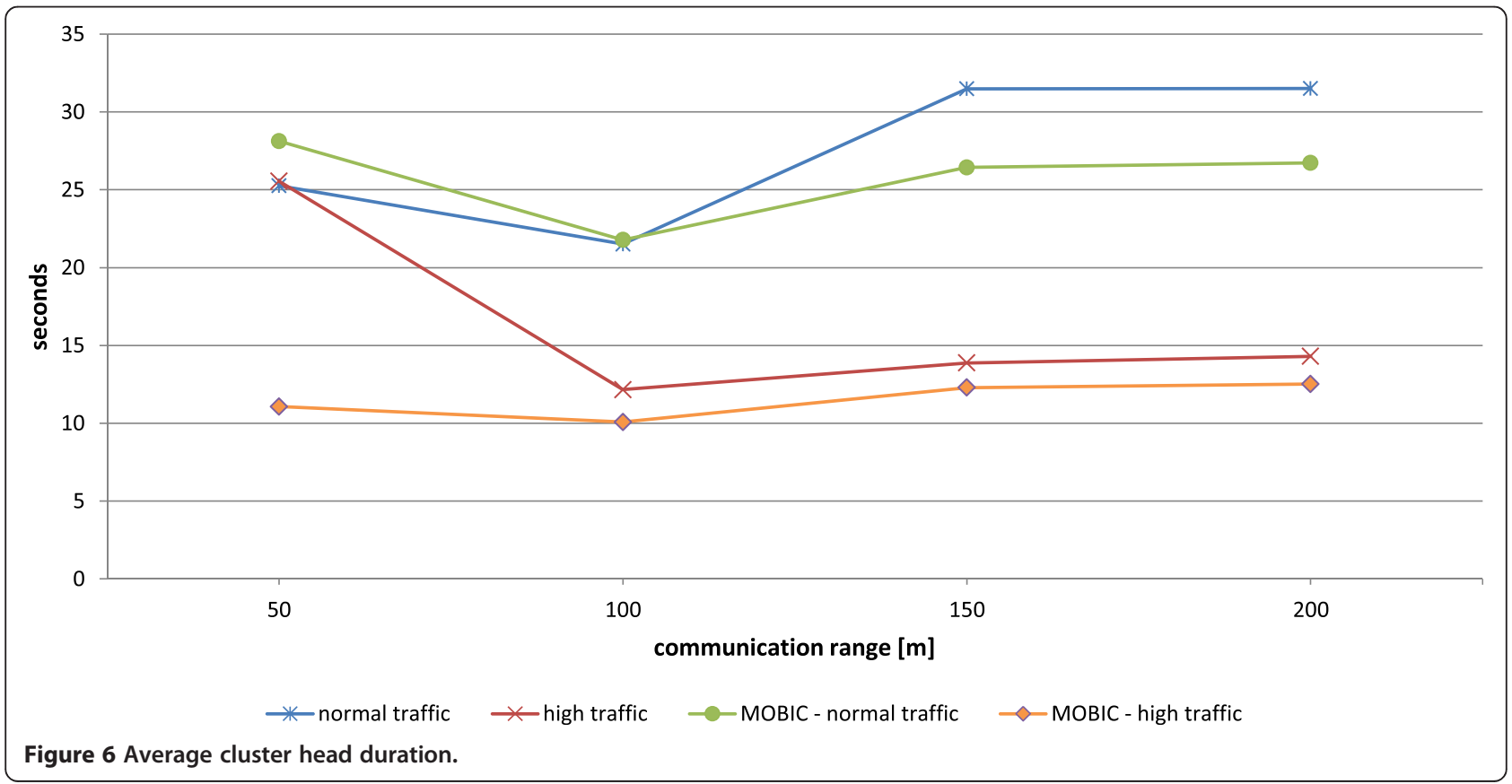




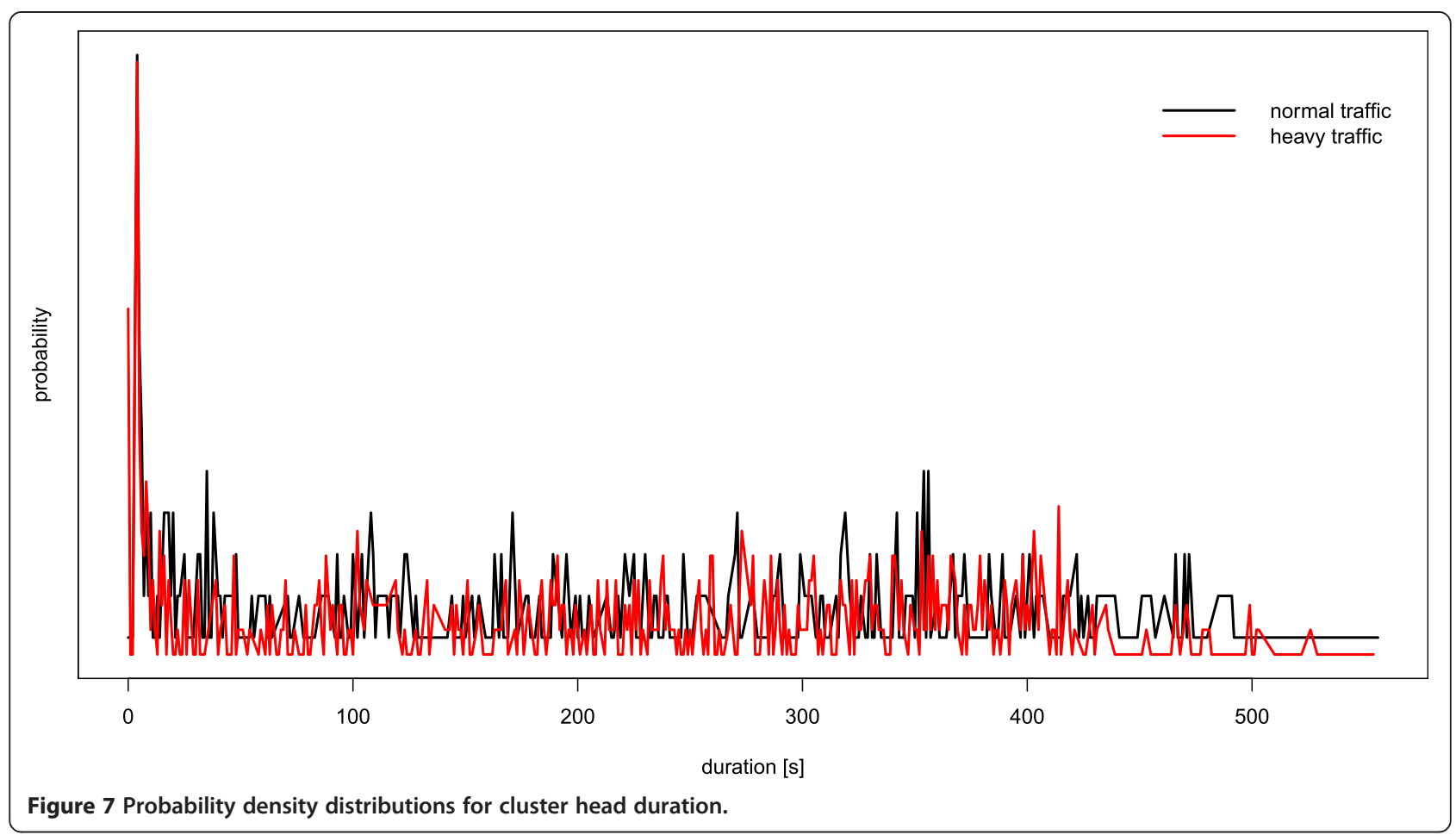

additionally concluded that role switches at a transmission range of $50 \mathrm{~m}$ follow the negative binomial distribution. For normal traffic conditions, we got a $p$ value of 0.94 ( $\operatorname{size}=2.41$, prob $=0.35$ ), and for heavily increased traffic conditions, we got a $p$ value of 0.02 ( $\operatorname{size}=3.43$, prob $=0.43)$. The rootogram of role switches in normal traffic conditions is shown in Figure 11. Unfortunately, role switches at longer transmission ranges could not be assigned to known distribution types at reasonable levels of significance. Nevertheless, role switches obtained in a

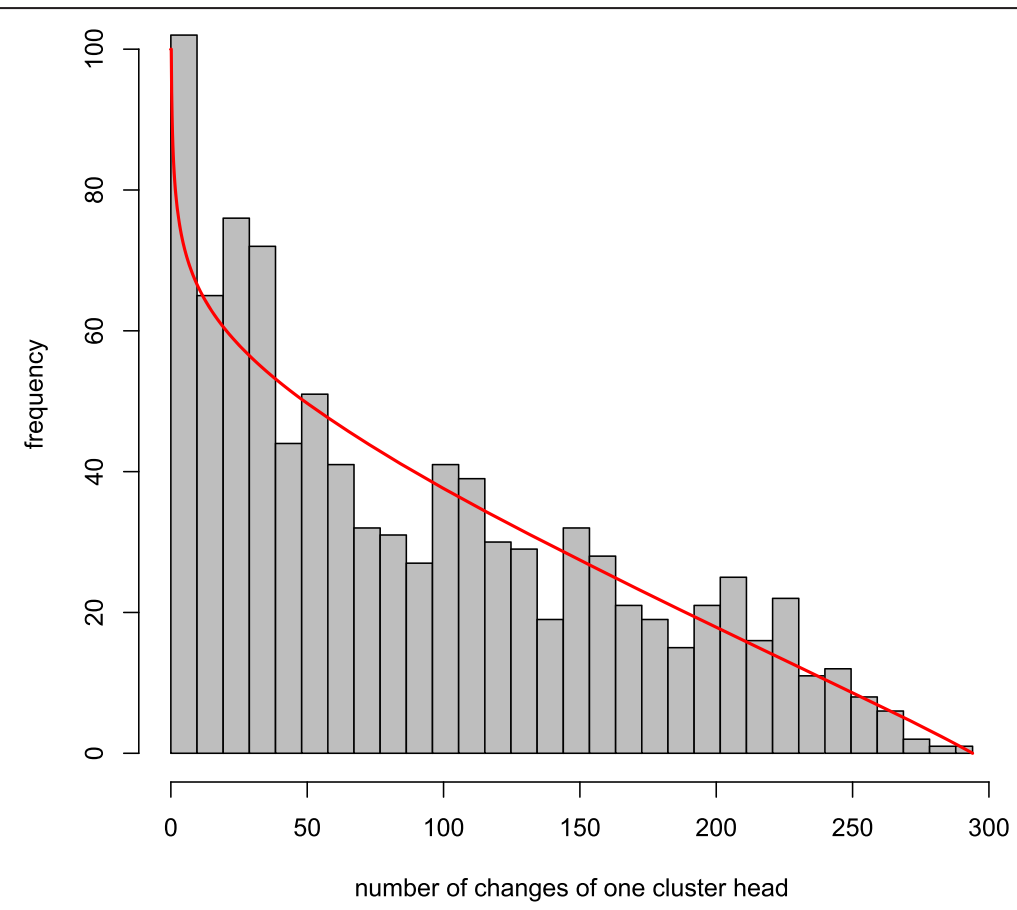

Figure 8 Histogram of changes of only one cluster head versus beta distribution. The histogram refers to heavily increased traffic conditions and a transmission range of $100 \mathrm{~m}$. 


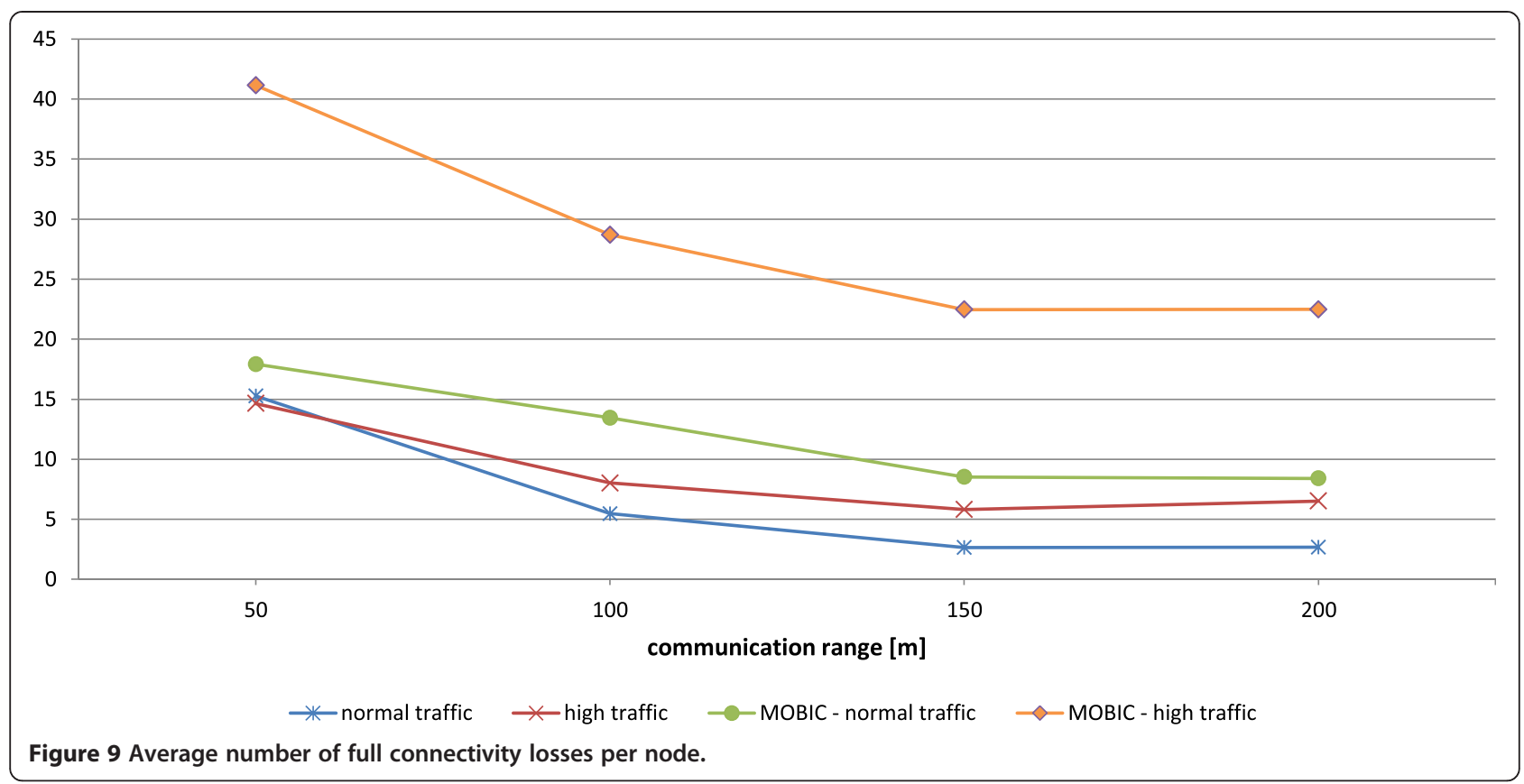

simulation of normal traffic conditions using the proposed clustering algorithm follow the same probability distribution as the ones obtained using the MOBIC algorithm. This may be claimed at a reasonable level of significance for all transmission ranges, since the Kolmogorov-Smirnov test gives $p$ values of $0.05,0.32$, $0.05,0.20$, and 0.20 , respectively. The histogram of role switches in normal traffic conditions and a transmission range of $150 \mathrm{~m}$ together with a distribution curve of role switches as obtained by the MOBIC algorithm are shown in Figure 12.

Figure 13 shows the average beacon size per node and relates to the protocol overhead. The measured values range from 104.95 to 106.02 bytes, proving the mathematical analysis of the protocol overhead where the calculated upper bound was determined as 107 bytes. The figure also shows that the connectivity increases with the communication range because a node is more likely

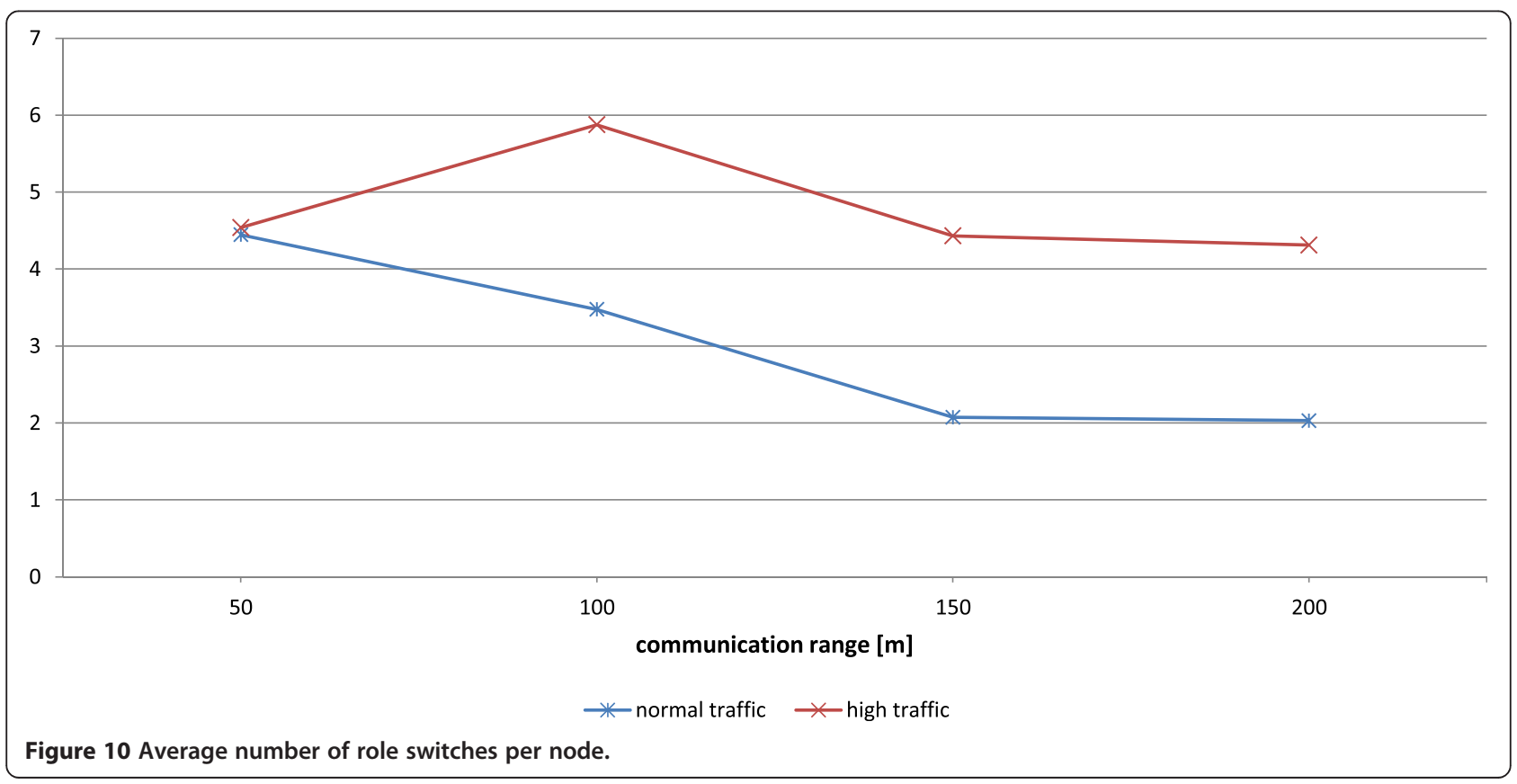




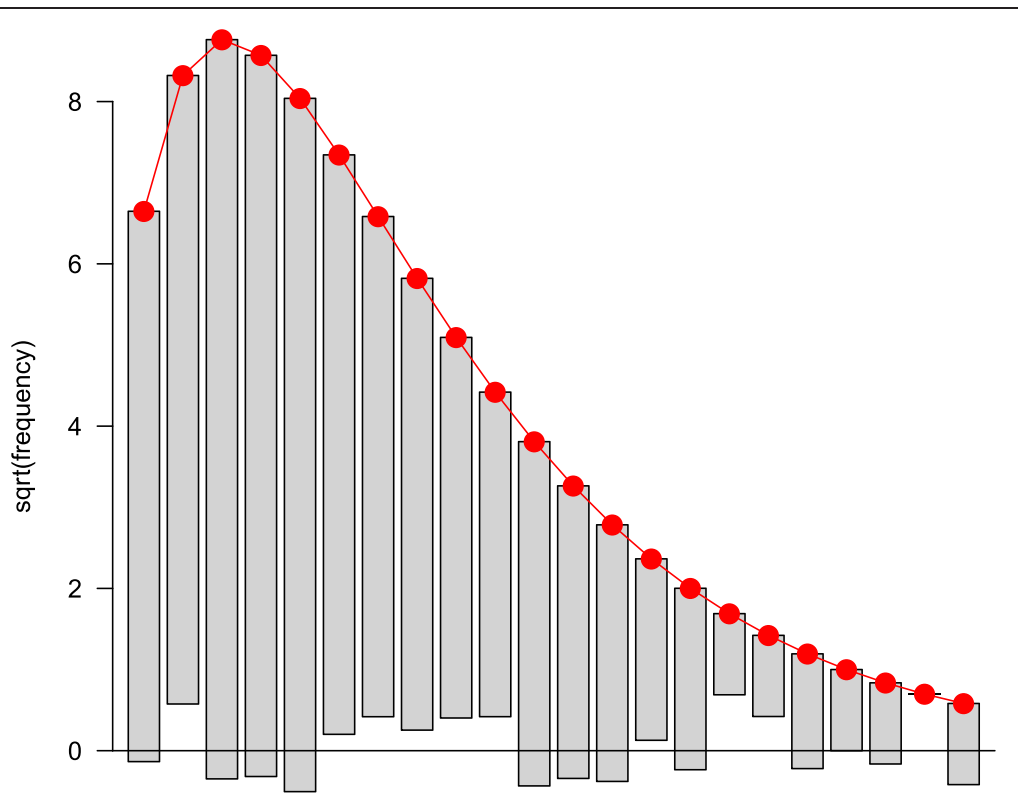

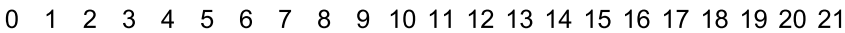

number of role switches

Figure 11 Rootogram of role switches versus negative binomial distribution. The rootogram refers to normal traffic conditions and a transmission range of $50 \mathrm{~m}$.

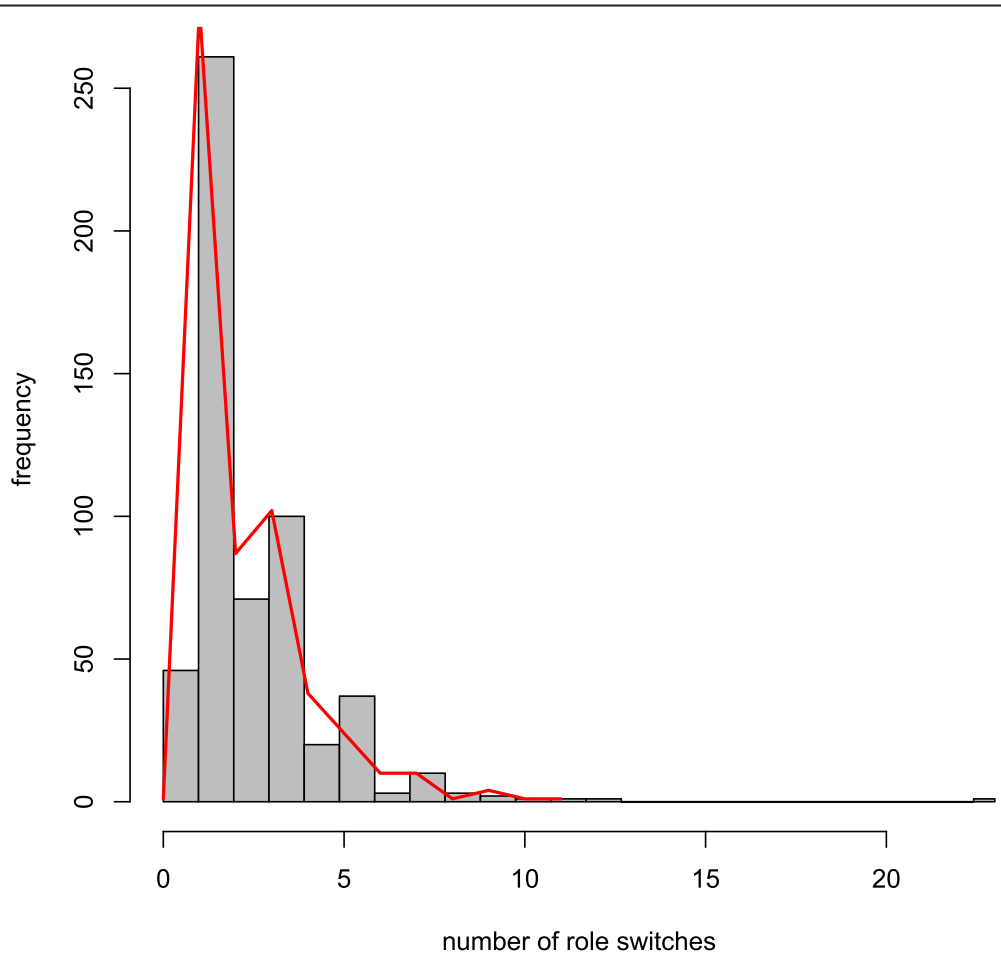

Figure 12 Histogram of role switches versus MOBIC distribution. The histogram refers to normal traffic conditions and a transmission range of $150 \mathrm{~m}$. 


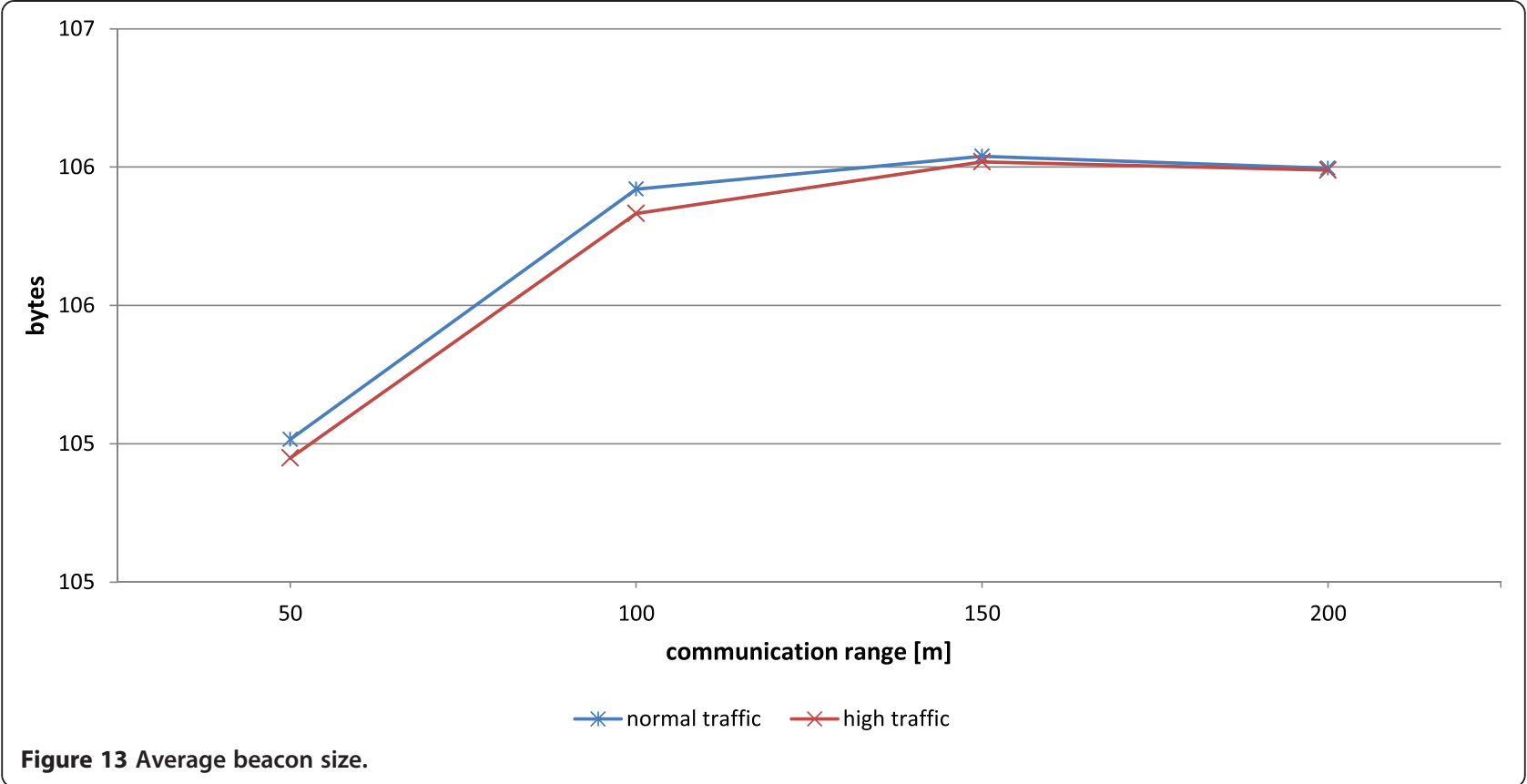

to find a suitable cluster head to connect to. For the beacon sizes, we may even claim that the samples are all drawn from the same population (the KolmogorovSmirnov test gives $p$ values larger than 0.31), no matter of the chosen transmission range or the type of the simulated traffic condition.

\section{Conclusions}

Clustering is a promising method for organizing vehicular wireless ad hoc networks to make the communication between vehicles more organized and efficient. Numerous different clustering algorithms have been proposed, striving to circumvent the high mobility of vehicles which negatively affect the communication abilities.

This paper presents a new clustering metric, named vehicle interconnection metric, which exposes the movement similarity of vehicles in the vicinity. It is based on beacon frames sent between vehicles and exposes the communication abilities between each pair of them, quickly adapting to the changes in the network. A clustering algorithm with dual cluster head connectivity and cluster head elimination instead of election, based on this metric, is also proposed. It strives to increase the connectivity and reduce the downtime when a node changes its cluster head, providing solid foundation for upper layer communication protocols.

Mathematic analysis shows that the proposed clustering algorithm requires very low memory and computing power. It exhibits moderate network overhead with limited upper bound, making it suitable for use even on congested roads. The simulation results prove the overhead analysis and show increased connectivity between vehicles, making the network more stable and disruption-free.

\section{Competing interests}

The authors declare that they have no competing interests.

\section{Acknowledgements}

The work was supported by the Ministry of Education, Science and Sport of Slovenia, the Slovenian Research Agency, and the Competence Center Open Communications Platform.

Received: 31 January 2014 Accepted: 7 October 2014 Published: 18 October 2014

\section{References}

1. Wikipedia, Dedicated short-range communications, Wikipedia, the free encyclopedia. (2012). http://en.wikipedia.org/wiki/Dedicated_short-range_communications. Accessed 13 April 2012

2. A Stern, A Kos, Povezljivost $v$ omrežjih VANET, Zbornik enaindvajsete mednarodne Elektrotehniške in računalniške konference ERK 2012 COMA (Portorož) 17-19 September 2012, pp. 53-56

3. S Vodopivec, J Bešter, A Kos, A Survey on Clustering Algorithms for Vehicular Ad-Hoc Networks, in 2012 35th International Conference on Telecommunications and Signal Processing (TSP), Prague, 3-7 July 2012, p. 5

4. P Basu, N Khan, TDC Little, A mobility based metric for clustering in mobile ad hoc networks, in 2001 International Conference on Distributed Computing Systems Workshop, Phoenix, April 2001, pp. 413-418

5. S Al-Sultan, M Al-Doori, A comprehensive survey on vehicular Ad Hoc network. J. Netw. Comput. Appl. 37, 380-392 (January 2014)

6. JA Volpe, Vulnerability assessment of the transportation infrastructure relying on the global positioning system. Nat. Transport. Syst. Center. (2001)

7. M Thomas, J Norton, A Jones, A Hopper, Global navigation space systems: reliance and vulnerabilities. R. Acad. Eng. (2011)

8. A Jafarnia-Jahromi, A Broumandan, J Nielsen, G Lachapelle, GPS vulnerability to spoofing threats and a review of antispoofing techniques. Int. J. Navigat. Observ. 2012, 1-16 (2012)

9. GPS World, Massive GPS jamming attack by North Korea. (2012). http://gpsworld.com/massive-gps-jamming-attack-by-north-korea/ 
10. Research and Innovative Technology Administration (RITA), ITS standards fact sheets, (2013). http://www.standards.its.dot.gov/Factsheets/Factsheet/80. Accessed 10 September 2014

11. W Li, A Tizghadam, A Leon-Garcia, Robust clustering for connected vehicles using local network criticality, in IEEE International Conference on Communications (ICC), 2012, Ottawa, 10-15 June 2012, pp. 7157-7161

12. B Hassanabadi, C Shea, L Zhang, S Valaee, Clustering in vehicular ad hoc networks using affinity propagation. Ad. Hoc. Netw. 13, 535-548 (February 2014)

13. S Ucar, SC Ergen, O Ozkasap, VMaSC: vehicular multi-hop algorithm for stable clustering in vehicular ad hoc networks 2013, in 2013 IEEE Wireless Communications and Networking Conference (WCNC), Shanghai, 7-10 April 2013, pp. 2381-2386

14. LA Maglaras, D Katsaros, Clustering in urban environments: virtual forces applied to vehicles, in Communications Workshops (ICC), 2013 IEEE International Conference on, Budapest, 9-13 June 2013, pp. 484-488

15. T Chung, W Cheng, Intelligent GPS-less speed detection and clustering in VANET, in 2012 Fourth International Conference on Ubiquitous and Future Networks, Phuket, 4-6 July 2012, pp. 145-150

16. KA Hafeez, L Zhao, Z Liao, BN-W Ma, A fuzzy-logic-based cluster head selection algorithm in VANETs, in 2012 IEEE International Conference on Communications (ICC), Ottawa, 10-15 June 2012, pp. 203-207

17. I Tal, G-M Muntean, User-oriented fuzzy logic-based clustering scheme for vehicular ad-hoc networks, in 2013 IEEE 77th Vehicular Technology Conference (VTC Spring), Dresden, 2-5 June 2013, pp. 1-5

18. H Idjmayyel, BR Qazi, JMH Elmirghani, Energy efficient double cluster head routing scheme in a city vehicular network, in 2013 27th International Conference on Advance Information Networking and Applications Workshops, Barcelona, 25-28 March 2013, pp. 1594-1599

19. A Louazani, SM Senouci, MA Bendaoud, Clustering-based algorithm for connectivity maintenance in vehicular ad-hoc networks, in 2014 14th International Conference on Innovations for Community Services (I4CS), Reims, 4-6 June 2014, pp. 34-38

20. R Chai, B Yang, L Li, Clustering-based data transmission algorithms for VANET, in 2013 International Conference on Wireless Communications \& Signal Processing (WCSP), Hangzhou, 24-26 October 2013, pp. 1-6

21. J Kponyo, Y Kuang, E Zhang, K Domenic, VANET cluster-on-demand minimum spanning tree (MST) Prim clustering algorithm, in 2013 International Conference on Computational Problem-solving (ICCP), Jiuzhai, 26-28 October 2013, pp. 101-104

22. F Chiti, R Fantacci, G Rigazzi, A mobility driven joint clustering and relay selection for IEEE 802.11p/WAVE vehicular networks, in 2014 IEEE International Conference on Communications (ICC), Sydney, 10-14 June 2014, pp. 348-353

23. T Stegel, J Sterle, U Sedlar, J Bešter, A Kos, SCTP multihoming provisioning in converged IP-based multimedia environment. Comput. Commun. 33(14), 1725-1735 (2010)

24. THenderson, M Lacage, G Riley, M Watrous, G Carneiro, T Pecorella, J Abraham, N Baldo, P Barnes, D Camara, Y Cheng, T Goff, S Jansen, D Lertpratchya, J Kopena, V Miletic, H Narra, J Pelkey, A Quereilhac, A Sacco, L Suresh, B Swenson, C Tapparello, M Weigle, ns-3. (2013). http://www.nsnam.org/. Accessed 11 April 2013

25. M Behrisch, L Bieker, J Erdmann, D Krajzewicz, SUMO - Simulation of Urban MObility: an overview, in SIMUL 2011, The Third International Conference on Advances in System Simulation, Barcelona, 23-29 October 2011, pp. 63-68

26. Y Pigné, G Danoy, P Bouvry, A platform for realistic online vehicular network management, in IEEE GLOBECOM Workshops, 2010. pp. 595-599

27. DC Montgomery, GC Runger, Applied Statistics and Probability for Engineers, 5th edn. (Wiley, USA, 2010)

doi:10.1186/1687-1499-2014-170

Cite this article as: Vodopivec et al: Vehicle interconnection metric and clustering protocol for improved connectivity in vehicular ad hoc networks. EURASIP Journal on Wireless Communications and Networking 2014 2014:170.

\section{Submit your manuscript to a SpringerOpen ${ }^{\circ}$ journal and benefit from:}

- Convenient online submission

- Rigorous peer review

- Immediate publication on acceptance

- Open access: articles freely available online

- High visibility within the field

- Retaining the copyright to your article

Submit your next manuscript at $\gg$ springeropen.com 